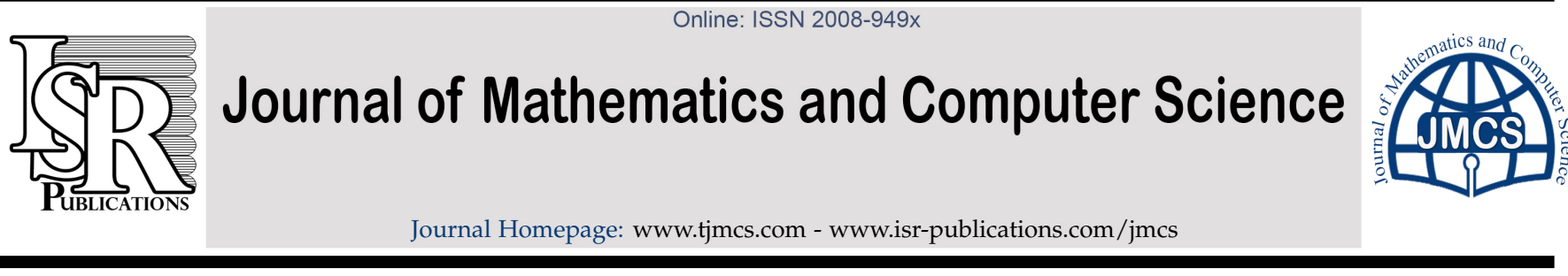

\title{
Multi-valued tripled fixed point results via CLR property in metric spaces with application
}

\author{
Muhammad Shoaiba ${ }^{a}$ Muhammad Sarwar ${ }^{\mathrm{a}, *}$, Yongjin Lib,* \\ ${ }^{a}$ Department of Mathematics, University of Malakand, Chakdara Dir(L), Pakistan. \\ ${ }^{b}$ Department of Mathematics Sun Yat-sen University, Guangzhou, Guangdong, China.
}

\begin{abstract}
In this work, using CLR property, tripled coincidence and common fixed point theorems for hybrid pair of mappings are studied. As an application, existence of solution to the system of integral equation is also discussed.
\end{abstract}

Keywords: Hybrid maps, tripled fixed point, CLR property.

(C)2018 All rights reserved.

\section{Introduction and preliminaries}

Alber and Gurre [2] presented weak contraction by generalizing the concept of contraction and showed the presence of fixed points for a self-map in Hilbert space. In [18], Rhoades proved that the result is still true for $\phi$-weak contraction in metric space. Dutta and Choudhury [9] investigated fixed point results by using $(\psi, \phi)$ weak contraction. In [22], Zhang and Song proved fixed point theorem for generalized $\phi-$ weak contraction for two self-maps. Doric [8] generalized the result of Zhang and Song for the presence of common fixed point with $(\psi, \phi)$ weak contraction.

Bhaskar and Lakshmikantham [10] derived some coupled fixed point results using mixed monotone property. Furthermore, they applied their results on a first order differential equation with periodic boundary conditions [11]. Lakshmikanthem and Ćirić [13] generalized the concept of mixed monotone mapping and established a coupled fixed point theorem for nonlinear contractions in partially ordered metric spaces.

Berinde and Borcut [5] introduced the concept of tripled fixed point for single-valued mappings in partially ordered metric spaces. Rao et al. [15] obtained a unique common triple fixed point theorem for hybrid pair of mappings in metric spaces. Aydi and Abbas [4] introduced the concept of $w$-compatiblity for single-valued tripled hybrid mappings.

\footnotetext{
${ }^{*}$ Corresponding author

Email addresses: shoaibkhanbs@yahoo.com (Muhammad Shoaib), sarwarswati@gamil.com (Muhammad Sarwar), stslyj@mail.sysu.edu.cn (Yongjin Li)
}

doi: $10.22436 /$ jmcs.018.02.04 
Deshpande et al. [7] introduced the concept of $w$-compatibility and weakly commutativity for hybrid pair of mappings, $\mathrm{F}: \Delta \times \Delta \times \Delta \rightarrow 2^{\Delta}$ and $\mathrm{g}: \Delta \rightarrow \Delta$ and established a common tripled fixed point theorem with generalized nonlinear contraction. In [6] the authors introduced the concept of E.A property and occasionally $w$-compatibility for hybrid pair $\mathrm{F}: \Delta \times \Delta \times \Delta \rightarrow 2^{\Delta}$ and $\mathrm{g}: \Delta \rightarrow \Delta$. Khan and Sumitra $[12,17]$ defined E.A and CLR property for coupled mapping. Rao et al. [16] defined CLRg property for multi-valued coupled maps in fuzzy metric spaces. For detail see $([3,20])$.

In the present manuscript, by using CLR property, some common hybrid tripled fixed point results for $(\psi, \phi)$ weak contraction in the setting of metric space are established. Throughout the paper $\mathbb{R}^{+}$, $\mathbb{N}$ and $\mathbb{N}_{0}$ stand for the set of all non-negative real numbers, the set of positive integers and the set of non-negative, integers respectively.

Definition 1.1. Suppose $\Delta$ is nonempty set and let $\mathrm{d}: \Delta \times \Delta \rightarrow \mathbb{R}^{+}$be a function satisfying the conditions:

1. $\mathrm{d}(\xi, \zeta)=0$ implies that $\xi=\zeta$, for all $\xi, \zeta \in \Delta$;

2. $\mathrm{d}(\xi, \zeta)>0$, for all $\xi \neq \zeta, \xi, \zeta \in \Delta$;

3. $\mathrm{d}(\xi, \zeta)=\mathrm{d}(\zeta, \xi)$, where $\xi, \zeta \in \Delta$;

4. $\mathrm{d}(\xi, \zeta) \leqslant \mathrm{d}(\xi, \tilde{z})+\mathrm{d}(\zeta, \tilde{z})$, for all $\xi, \zeta, \tilde{z} \in \Delta$.

Then $\mathrm{d}$ is a metric on $\Delta$ and the pair $(\Delta, \mathrm{d})$ is called metric space.

Definition 1.2 ([19]). Let $\Delta$ be a nonempty set and $F: \Delta \times \Delta \times \Delta \rightarrow \Delta$ be a given mapping. An element $(x, y, z) \in \Delta \times \Delta \times \Delta$ is called a tripled fixed point of the mapping $F$ if $F(x, y, z)=x, F(y, z, x)=y$ and $\mathrm{F}(z, x, y)=z$.

We denote the set of tripled coincidence points of mappings $F$ and $g$ by $C(F, g)$. Note that if $(x, y, z) \in$ $C(F, g)$, then $(y, z, x)$ and $(z, x, y)$ are also in $C(F, g)$.

Definition 1.3 ([7]). Let $\mathrm{F}: \Delta \times \Delta \times \Delta \rightarrow 2^{\Delta}$ be a multi-valued mapping and $\mathrm{g}$ be a self-map on $\Delta$. The hybrid pair $(F, g)$ is called $w$-compatible if $g(F(x, y, z)) \in F(g x, g y, g z)$ whenever $(x, y, z) \in C(F, g)$.

Definition 1.4 ([7]). Let $\mathrm{F}: \Delta \times \Delta \times \Delta \rightarrow 2^{\Delta}$ be a multi-valued mapping and $\mathrm{g}$ be a self-map on $\Delta$. The mapping $g$ is called F-weakly commuting at some point $(x, y, z) \in \Delta^{3}$, if $g^{2} x \in F(g x, g y, g z), g^{2} y \in$ $\mathrm{F}(\mathrm{gy}, \mathrm{gz}, \mathrm{gx})$ and $\mathrm{g}^{2} z \in \mathrm{F}(\mathrm{gz}, \mathrm{gx}, \mathrm{gy})$.

Definition 1.5 ([7]). Let $\Delta$ be a nonempty set, $\mathrm{F}: \Delta \times \Delta \times \Delta \rightarrow 2^{\Delta}$ (a collection of all nonempty subsets of $\Delta)$ and $g$ be a self-map on $\Delta$. An element $(x, y, z) \in \Delta \times \Delta \times \Delta$ is called

(1) a tripled fixed point of $F$ if $x \in F(x, y, z), y \in F(y, z, x)$ and $z \in F(z, x, y)$;

(2) a tripled coincidence point of hybrid pair $\{F, g\}$ if $g(x) \in F(x, y, z), g(y) \in F(y, z, x)$ and $g(z) \in$ $\mathrm{F}(z, x, y)$;

(3) a common tripled fixed point of hybrid pair $F, g$ if $x=g(x) \in F(x, y, z), y=g(y) \in F(y, z, x)$ and $z=\mathrm{g}(z) \in \mathrm{F}(z, x, y)$.

Definition 1.6 ([21]). Maps $f, g: \Delta \rightarrow \Delta$ are said to satisfy the common limit in the range of $f$ with respect to $g$ (shortly, the $\left(C L R_{f}\right)$-property with respect to $g$ ) if there exists a sequence $\xi_{n}$ in $\Delta$ such that for some $u \in \Delta, \lim _{n \rightarrow \infty} f \xi_{n}=\lim _{n \rightarrow \infty} g \xi_{n}=f u$.

The following definition can be found in [1].

Definition 1.7. Mappings $f: \Delta \rightarrow \Delta, S: \Delta \rightarrow \mathrm{CB}(\Delta)$ on metric space $(\Delta, \mathrm{d})$ are said to satisfy the common limit in the range of $f$ with respect to $S$ (shortly, the $\left(C L R_{f}\right)$-property with respect to $S$ ) if there exists a sequence $\xi_{n}$ in $\Delta$ and $\Omega_{1} \in \mathrm{CB}(\Delta)$ such that for some $u \in \Delta, \lim _{n \rightarrow \infty} f \xi_{n}=f u \in \Omega_{1}=\lim _{n \rightarrow \infty} S \xi_{n}=f u$. 
Definition 1.8. Mappings $f, g: \Delta \rightarrow \Delta$ and $S, T: \Delta \rightarrow C B(\Delta)$ on metric space $(\Delta, d)$ are to satisfy the common limit in the range of $f$ with respect to $S$ (shortly, the $\left(C L R_{f}\right)$-property with respect to $S$ ) if there exist sequences $\xi_{n}$ and $\zeta_{n}$ in $\Delta$ and $\Omega_{1}, \Omega_{2} \in \mathrm{CB}(\Delta)$ such that for some $u \in \Delta, \lim _{n \rightarrow \infty} S \xi_{n}=\Omega_{1}$, $\lim _{n \rightarrow \infty} T \zeta_{n}=\Omega_{2}$ and $\lim _{n \rightarrow \infty} f \xi_{n}=\lim _{n \rightarrow \infty} g \zeta_{n}=f u \in \Omega_{1} \cap \Omega_{2}$.

Definition 1.9. Mappings $f: \Delta \rightarrow \Delta$ and $S: \Delta \times \Delta \rightarrow \mathrm{CB}(\Delta)$ on metric space $(\Delta, \mathrm{d})$ are said to have E.A property if there exist sequences $\xi_{n}$ and $\zeta_{n}$ in $\Delta$ and $\Omega_{1}, \Omega_{2} \in \operatorname{CB}(\Delta)$ such that for some $u \in \Delta$, $\lim _{n \rightarrow \infty} f \xi_{n}=u \in \Omega_{1}=\lim _{n \rightarrow \infty} S\left(\xi_{n}, \zeta_{n}\right), \lim _{n \rightarrow \infty} f \zeta_{n}=v \in \Omega_{2}=\lim _{n \rightarrow \infty} S\left(\zeta_{n}, \xi_{n}\right)$.

Definition 1.10. Mappings $f: \Delta \rightarrow \Delta$ and $S: \Delta \times \Delta \rightarrow \mathrm{CB}(\Delta)$ on metric space $(\Delta, \mathrm{d})$ are to satisfy the common limit in the range of $f$ with respect to $S$ (shortly, the $\left(C L R_{f}\right)$-property with respect to $S$ ) if there exist sequences $\xi_{n}$ and $\zeta_{n}$ in $\Delta$ and $\Omega_{1}, \Omega_{2} \in \operatorname{CB}(\Delta)$ such that for some $u \in \Delta$

$$
\begin{aligned}
& \lim _{n \rightarrow \infty} f \xi_{n}=u=f \xi \in \Omega_{1}=\lim _{n \rightarrow \infty} S\left(\xi_{n}, \zeta_{n}\right), \\
& \lim _{n \rightarrow \infty} f \zeta_{n}=v=f \zeta \in \Omega_{2}=\lim _{n \rightarrow \infty} S\left(\zeta_{n}, \xi_{n}\right) .
\end{aligned}
$$

In the sequel we also need the following:

Assume that $\mathrm{d}$ is a metric on $\Delta$, define the function $\mathrm{H}: \mathrm{CB}(\Delta) \times \mathrm{CB}(\Delta) \rightarrow \mathbb{R}^{+}$for $\Omega_{1}, \Omega_{2} \in \mathrm{CB}(\Delta)$ by

$$
\mathrm{H}\left(\Omega_{1}, \Omega_{2}\right)=\max \left\{\sup _{\sigma \in \Omega_{1}} d\left(\sigma, \Omega_{2}\right), \sup _{\zeta \in \Omega_{1}} d\left(\zeta, \Omega_{1}\right)\right\},
$$

where

$$
\begin{aligned}
\mathrm{d}\left(\xi, \Omega_{1}\right) & =\inf \left\{\mathrm{d}(\xi, \zeta): \zeta \in \Omega_{1}\right\}, \\
\delta\left(\Omega_{1}, \Omega_{2}\right) & =\sup \left\{\mathrm{d}(\sigma, \zeta): \sigma \in \Omega_{1}, \zeta \in \Omega_{2}\right\}
\end{aligned}
$$

and

$$
\mathrm{D}\left(\Omega_{1}, \Omega_{2}\right)=\inf \left\{\mathrm{d}(\sigma, \zeta): \sigma \in \Omega_{1}, \zeta \in \Omega_{2}\right\} .
$$

The following can be deduced from the definition of $\delta$

$$
\begin{aligned}
& \delta\left(\Omega_{1}, \Omega_{2}\right)=\delta\left(\Omega_{2}, \Omega_{1}\right), \\
& \delta\left(\Omega_{1}, \Omega_{3}\right) \leqslant \delta\left(\Omega_{1}, \Omega_{2}\right)+\delta\left(\Omega_{2}, \Omega_{3}\right), \\
& \delta\left(\Omega_{1}, \Omega_{2}\right)=0 \Leftrightarrow \Omega_{1}=\Omega_{2}=\{\sigma\}, \\
& \delta\left(\Omega_{1}, \Omega_{1}\right)=\operatorname{diam} \Omega_{1} .
\end{aligned}
$$

Lemma 1.11 ([14]). Let $(\Delta, \mathrm{d})$ be a metric space. For any $\Omega_{1}, \Omega_{2}, \Omega_{3} \in \mathrm{CB}(\Delta)$ and $\xi, \zeta \in \Delta$, the following hold:

1. $\mathrm{d}\left(\xi, \Omega_{2}\right) \leqslant \mathrm{d}(\xi, \zeta), \forall \zeta \in \Omega_{2}$;

2. $\delta\left(\Omega_{1}, \Omega_{2}\right) \leqslant H\left(\Omega_{1}, \Omega_{2}\right)$;

3. $\mathrm{d}\left(\xi, \Omega_{2}\right) \leqslant \mathrm{H}\left(\Omega_{1}, \Omega_{2}\right), \forall \xi \in \Omega_{1}$;

4. $\mathrm{H}\left(\Omega_{1}, \Omega_{1}\right)=0$;

5. $\mathrm{H}\left(\Omega_{1}, \Omega_{2}\right)=\mathrm{H}\left(\Omega_{2}, \Omega_{1}\right)$;

6. $H\left(\Omega_{1}, \Omega_{3}\right) \leqslant H\left(\Omega_{1}, \Omega_{2}\right)+H\left(\Omega_{2}, \Omega_{3}\right)$;

7. $H\left(\xi, \Omega_{1}\right) \leqslant d(\xi, \zeta)+d\left(\zeta, \Omega_{1}\right)$.

Lemma 1.12 ([14]). Suppose $(\Delta, \mathrm{d})$ is metric space. Furthermore, let $\Omega_{1}, \Omega_{2} \in \mathrm{CB}(\Delta)$, then for every $\mathrm{h}>1$ and for each $\sigma \in \Omega_{1}$ there exists $\zeta(\sigma) \in \Omega_{2}$ such that $\mathrm{d}(\sigma, \zeta) \leqslant \mathrm{hH}\left(\Omega_{1}, \Omega_{2}\right)$.

In [14] it is shown that the above lemma is also true for $h \geqslant 1$.

Lemma 1.13. Assume a metric space $(\Delta, \mathrm{d})$. Let $\Omega_{1}, \Omega_{2} \in \mathrm{CB}(\Delta)$, then for every $\mathrm{h} \geqslant 1$ and for each $\sigma \in \Omega_{1}$ there exists $\zeta(\sigma) \in \Omega_{2}$ such that $\mathrm{d}(\sigma, \zeta) \leqslant \mathrm{hH}\left(\Omega_{1}, \Omega_{2}\right)$. 
Definition 1.14. Let $f: \Delta \rightarrow \Delta, \mathrm{F}: \Delta \times \Delta \times \Delta \rightarrow \mathrm{Cl}(\Delta)$ on metric space $(\Delta, \mathrm{d})$. Mappings $(\mathrm{F}, \mathrm{f})$ have $\left(\mathrm{CLR}_{\mathrm{f}}\right)$-property, if there exist sequences $x_{n}, y_{n}, z_{n}$ in $\Delta$ for some $z_{1}, z_{2}, z_{3} \in \Delta$ and $C_{1}, D_{1}, E_{1} \in C B(\Delta)$ such that

$$
\begin{aligned}
& \lim _{n \rightarrow \infty} F\left(x_{n}, y_{n}, z_{n}\right)=C_{1}, \lim _{n \rightarrow \infty} f x_{n}=f z_{1} \in C_{1}, \\
& \lim _{n \rightarrow \infty} F\left(y_{n}, x_{n}, z_{n}\right)=D_{1}, \lim _{n \rightarrow \infty} f y_{n}=f z_{2} \in D_{1}, \\
& \lim _{n \rightarrow \infty} F\left(z_{n}, y_{n}, x_{n}\right)=E_{1}, \lim _{n \rightarrow \infty} f z_{n}=f z_{3} \in E_{1} .
\end{aligned}
$$

Definition 1.15. Let $\mathrm{f}, \mathrm{g}: \Delta \rightarrow \Delta, \mathrm{F}, \mathrm{G}: \Delta \times \Delta \times \Delta \rightarrow \mathrm{Cl}(\Delta)$ on metric space $(\Delta, \mathrm{d})$. Mappings $(\mathrm{F}, \mathrm{f})$ and $(G, g)$ have $\left(C L R_{f}\right)$ and $\left(C L R_{g}\right)$-property, respectively, if there exist sequences $x_{n}, y_{n}, z_{n}$ and $u_{n} v_{n}, w_{n}$ in $\Delta$ for some $z_{1}, z_{2}, z_{3} \in \Delta$ and $\mathrm{C}_{1}, \mathrm{C}_{2}, \mathrm{D}_{1}, \mathrm{D}_{2}, \mathrm{E}_{1}, \mathrm{E}_{2} \in \mathrm{CB}(\Delta)$ such that

$$
\begin{aligned}
& \lim _{n \rightarrow \infty} F\left(x_{n}, y_{n}, z_{n}\right)=C_{1}, \lim _{n \rightarrow \infty} G\left(u_{n}, v_{n}, w_{n}\right)=C_{2}, \\
& \lim _{n \rightarrow \infty} f x_{n}=f z_{1} \in C_{1}, \lim _{n \rightarrow \infty} g u_{n}=g z_{1} \in C_{2}, \\
& \lim _{n \rightarrow \infty} F\left(y_{n}, x_{n}, z_{n}\right)=D_{1}, \lim _{n \rightarrow \infty} G\left(v_{n}, u_{n}, w_{n}\right)=D_{2}, \\
& \lim _{n \rightarrow \infty} f y_{n}=f z_{2} \in D_{1}, \lim _{n \rightarrow \infty} g v_{n}=g z_{2} \in D_{2}, \\
& \lim _{n \rightarrow \infty} F\left(z_{n}, y_{n}, x_{n}\right)=E_{1}, \lim _{n \rightarrow \infty} G\left(w_{n}, v_{n}, u_{n}\right)=E_{2}, \\
& \lim _{n \rightarrow \infty} f z_{n}=f z_{3} \in E_{1}, \lim _{n \rightarrow \infty} g w_{n}=g z_{3} \in E_{2} .
\end{aligned}
$$

\section{Main results}

In our main results we use the following two classes.

$$
\begin{aligned}
& \Psi=\left\{\begin{array}{l}
\psi: \mathbb{R}^{+} \rightarrow \mathbb{R}^{+} \text {is continuous and non-decreasing function such that } \\
\psi(\tau)=0 \text { if and only if } \tau=0 .
\end{array}\right\}, \\
& \Phi=\left\{\begin{array}{l}
\phi: \mathbb{R}^{+} \rightarrow \mathbb{R}^{+} \text {is a lower semicontinuous and non-decreasing } \\
\text { function such that } \phi(\tau)=0 \text { if and only if } \tau=0
\end{array}\right\} .
\end{aligned}
$$

Theorem 2.1. Let $\mathrm{f}, \mathrm{g}: \Delta \rightarrow \Delta, \mathrm{F}, \mathrm{G}: \Delta \times \Delta \times \Delta \rightarrow \mathrm{Cl}(\Delta)$ on metric space $(\Delta, \mathrm{d})$. Furthermore assume that $(\mathrm{F}, \mathrm{f})$ have $\mathrm{CLR}_{\mathrm{f}}$ and $(\mathrm{G}, \mathrm{g})$ have $\mathrm{CLR}_{\mathrm{g}}$-property, also the following condition holds:

$$
\psi\left(H^{p}(F(x, y, z), G(u, v, w))\right) \leqslant \psi(\Theta(x, y, z, u, v, w))-\phi(\Theta(x, y, z, u, v, w)),
$$

where

$$
\Theta(x, y, z, u, v, w)=\max \left\{\begin{array}{l}
d^{p}(f x, g u), d^{p}(f x, F(x, y, z)), d^{p}(g u, G(u, v, w)), \\
d^{p}(f y, g v), d^{p}(f y, F(y, z, x)), d^{p}(g v, G(v, w, u)), \\
d^{p}(f z, g w), d^{p}(f z, F(z, x, y)), d^{p}(g w, G(w, u, v)), \\
\frac{d^{p}(f x, g u)+d^{p}(f x, F(x, y, z))+d^{p}(g u, G(u, v, w))}{2}, \\
\frac{d^{p}(f y, g v)+d^{p}(f y, F(y, z, x))+d^{p}(g v, G(v, w, u))}{2}, \\
\frac{d^{p}(f z, g w)+d^{p}(f z, F(z, x, y))+d^{p}(g w, G(w, u, v))}{2} .
\end{array}\right\} .
$$

Here, $\psi \in \Psi, \phi \in \Phi$ and $p \geqslant 1$. Then

$\left(\mathrm{A}_{1}\right)(\mathrm{f}, \mathrm{F})$ have tripled coincidence point.

$\left(\mathrm{A}_{2}\right)(\mathrm{g}, \mathrm{G})$ have tripled coincidence point.

$\left(\mathrm{A}_{3}\right)$ If $\mathrm{f}$ is F-weakly commuting for $\left(z_{1}, z_{2}, z_{3}\right) \in \mathrm{C}(\mathrm{F}, \mathrm{f})$ and $\mathrm{f}^{2} z_{1}=\mathrm{f} z_{1}, \mathrm{f}^{2} z_{2}=\mathrm{f} z_{2}, \mathrm{f}^{2} z_{3}=\mathrm{f} z_{3}$, then $\mathrm{F}$ and $\mathrm{f}$ have a common tripled fixed point.

$\left(\mathrm{A}_{4}\right)$ If $\mathrm{g}$ is G-weakly commuting for $\left(w_{1}, w_{2}, w_{3}\right) \in \mathrm{C}(\mathrm{G}, \mathrm{g})$ and $\mathrm{g}^{2} w_{1}=g w_{1}, g^{2} w_{2}=g w_{2}, g^{2} w_{3}=g w_{3}$, then $\mathrm{G}$ and $\mathrm{g}$ have a common tripled fixed point. 
$\left(A_{5}\right) F, G, f$ and $g$ have common tripled fixed point if $\left(A_{3}\right)$ and $\left(A_{4}\right)$ hold.

Proof. Since $(F, f)$ have $\left(C L R_{f}\right)$-property, there exist sequences $x_{n}, y_{n}, z_{n}$ and $C_{1}, D_{1}, E_{1} \in C B(\Delta)$ such that

$$
\begin{array}{ll}
\lim _{n \rightarrow \infty} F\left(x_{n}, y_{n}, z_{n}\right)=C_{1}, & \lim _{n \rightarrow \infty} f x_{n}=f z_{1} \in C_{1}, \\
\lim _{n \rightarrow \infty} F\left(y_{n}, x_{n}, z_{n}\right)=D_{1}, & \lim _{n \rightarrow \infty} f y_{n}=f z_{2} \in D_{1}, \\
\lim _{n \rightarrow \infty} F\left(z_{n}, y_{n}, x_{n}\right)=E_{1}, & \lim _{n \rightarrow \infty} f z_{n}=f z_{3} \in E_{1},
\end{array}
$$

for some $z_{1}, z_{2}, z_{3} \in \Delta$.

Similarly, since $(G, g)$ have $\left(C_{L} g\right)$-property, there exist sequences $u_{n}, v_{n}, w_{n}$ and $C_{2}, D_{2}, E_{2} \in C B(\Delta)$ such that

$$
\begin{array}{ll}
\lim _{n \rightarrow \infty} \mathrm{G}\left(\mathrm{u}_{n}, v_{n}, w_{n}\right)=\mathrm{C}_{2}, & \lim _{n \rightarrow \infty} \mathrm{g} \mathrm{u}_{\mathrm{n}}=\mathrm{g} z_{1} \in \mathrm{C}_{2}, \\
\lim _{n \rightarrow \infty} \mathrm{G}\left(v_{n}, \mathrm{u}_{n}, w_{n}\right)=\mathrm{D}_{2}, & \lim _{n \rightarrow \infty} \mathrm{g} v_{n}=\mathrm{g} z_{2} \in \mathrm{D}_{2}, \\
\lim _{n \rightarrow \infty} \mathrm{G}\left(w_{n}, v_{n}, u_{n}\right)=E_{2}, & \lim _{n \rightarrow \infty} g w_{n}=g z_{3} \in E_{2},
\end{array}
$$

for some $z_{1}, z_{2}, z_{3} \in \Delta$.

Putting $x=x_{n}, y=y_{n}, z=z_{n}$ and $u=u_{n}, v=v_{n}, w=w_{n}$ in inequality (2.1) we get

$\psi\left(H^{p}\left(F\left(x_{n}, y_{n}, z_{n}\right), G\left(u_{n}, v_{n}, w_{n}\right)\right)\right) \leqslant \psi\left(\Theta\left(x_{n}, y_{n}, z_{n}, u_{n}, v_{n}, w_{n}\right)\right)-\phi\left(\Theta\left(x_{n}, y_{n}, z_{n}, u_{n}, v_{n}, w_{n}\right)\right)$,

where

$$
\Theta\left(x_{n}, y_{n}, z_{n}, u_{n}, v_{n}, w_{n}\right)=\max \left\{\begin{array}{l}
d^{p}\left(f x_{n}, g u_{n}\right), d^{p}\left(f x_{n}, F\left(x_{n}, y_{n}, z_{n}\right)\right), d^{p}\left(g u_{n}, G\left(u_{n}, v_{n}, w_{n}\right)\right), \\
d^{p}\left(f y_{n}, g v_{n}\right), d^{p}\left(f y_{n}, F\left(y_{n}, z_{n}, x_{n}\right)\right), d^{p}\left(g v_{n}, G\left(v_{n}, w_{n}, u_{n}\right)\right), \\
d^{p}\left(f z_{n}, g w_{n}\right), d^{p}\left(f z, F\left(z_{n}, x_{n}, y_{n}\right)\right), d^{p}\left(g w_{n}, G\left(w_{n}, u_{n}, v_{n}\right)\right), \\
\frac{d^{p}\left(f x_{n}, g u_{n}\right)+d^{p}\left(f x, F\left(x_{n}, y_{n}, z_{n}\right)\right)+d^{p}\left(g u_{n}, G\left(u_{n}, v_{n}, w_{n}\right)\right)}{2}, \\
\frac{d^{p}\left(f y_{n}, g v_{n}\right)+d^{p}\left(f y, F\left(y_{n}, z_{n}, x_{n}\right)\right)+d^{p}\left(g v_{n}, G\left(v_{n}, w_{n}, u_{n}\right)\right)}{2}, \\
\frac{d^{p}\left(f z_{n}, g w_{n}\right)+d^{p}\left(f z_{n}, F\left(z_{n}, x_{n}, y_{n}\right)\right)+d^{p}\left(g w_{n}, G\left(w_{n}, u_{n}, v_{n}\right)\right)}{2} .
\end{array}\right\} .
$$

Applying limit to (2.2) we have

$$
\begin{aligned}
\lim _{n \rightarrow \infty} \psi\left(H^{p}\left(F\left(x_{n}, y_{n}, z_{n}\right), G\left(u_{n}, v_{n}, w_{n}\right)\right)\right) \leqslant & \lim _{n \rightarrow \infty} \psi\left(\Theta\left(x_{n}, y_{n}, z_{n}, u_{n}, v_{n}, w_{n}\right)\right) \\
& -\lim _{n \rightarrow \infty} \phi\left(\Theta\left(x_{n}, y_{n}, z_{n}, u_{n}, v_{n}, w_{n}\right)\right),
\end{aligned}
$$

where

$$
\lim _{n \rightarrow \infty} \Theta\left(x_{n}, y_{n}, z_{n}, u_{n}, v_{n}, w_{n}\right)=\max \left\{d^{p}\left(f z_{1}, g z_{1}\right), d^{p}\left(f z_{2}, g z_{2}\right), d^{p}\left(f z_{3}, g z_{3}\right)\right\},
$$

which implies

$$
\psi\left(d^{p}\left(f z_{1}, g z_{1}\right)\right) \leqslant \psi\left(\max \left\{\begin{array}{l}
d^{p}\left(f z_{1}, g z_{1}\right), \\
d^{p}\left(f z_{2}, g z_{2}\right), \\
d^{p}\left(f z_{3}, g z_{3}\right)
\end{array}\right\}\right)-\phi\left(\max \left\{\begin{array}{l}
d^{p}\left(f z_{1}, g z_{1}\right), \\
d^{p}\left(f z_{2}, g z_{2}\right), \\
d^{p}\left(f z_{3}, g z_{3}\right)
\end{array}\right\}\right) .
$$

By putting $x=y_{n}, y=z_{n}, z=x_{n}$ and $u=v_{n}, v=w_{n}, w=u_{n}$ in inequality (2.1) we get

$$
\psi\left(H^{p}\left(F\left(x_{n}, y_{n}, z_{n}\right), G\left(u_{n}, v_{n}, w_{n}\right)\right)\right) \leqslant \psi\left(\Theta\left(x_{n}, y_{n}, z_{n}, u_{n}, v_{n}, w_{n}\right)\right)-\phi\left(\Theta\left(x_{n}, y_{n}, z_{n}, u_{n}, v_{n}, w_{n}\right)\right) .
$$

By applying limit to (2.4) we conclude that

$$
\psi\left(d^{p}\left(f z_{2}, g z_{2}\right)\right) \leqslant \psi\left(\max \left\{\begin{array}{l}
d^{p}\left(f z_{1}, g z_{1}\right), \\
d^{p}\left(f z_{2}, g z_{2}\right), \\
d^{p}\left(f z_{3}, g z_{3}\right)
\end{array}\right\}\right)-\phi\left(\max \left\{\begin{array}{l}
d^{p}\left(f z_{1}, g z_{1}\right), \\
d^{p}\left(f z_{2}, g z_{2}\right), \\
\left.d^{p}\left(f z_{3}, g z_{3}\right)\right)
\end{array}\right\}\right) .
$$


Next, putting $x=z_{n}, y=y_{n}, z=x_{n}$ and $u=w_{n}, v=v_{n}, w=u_{n}$ in inequality (2.1) we get

$\psi\left(H^{p}\left(F\left(z_{n}, y_{n}, x_{n}\right), G\left(w_{n}, v_{n}, u_{n}\right)\right) \leqslant \psi\left(\Theta\left(z_{n}, y_{n}, x_{n}, w_{n}, v_{n}, u_{n}\right)\right)-\phi\left(\Theta\left(z_{n}, y_{n}, x_{n}, w_{n}, v_{n}, u_{n}\right)\right)\right.$.

Again, applying limit to (2.6), by simplification one can write

$$
\psi\left(d^{p}\left(f z_{3}, g z_{3}\right)\right) \leqslant \psi\left(\max \left\{\begin{array}{l}
d^{p}\left(f z_{1}, g z_{1}\right), \\
d^{p}\left(f z_{2}, g z_{2}\right), \\
d^{p}\left(f z_{3}, g z_{3}\right)
\end{array}\right\}\right)-\phi\left(\max \left\{\begin{array}{l}
d^{p}\left(f z_{1}, g z_{1}\right), \\
d^{p}\left(f z_{2}, g z_{2}\right), \\
d^{p}\left(f z_{3}, g z_{3}\right)
\end{array}\right\}\right) .
$$

Combining (2.3), (2.5) and (2.7) we have

$$
\psi\left(\max \left\{\begin{array}{l}
d^{p}\left(f z_{1}, g z_{1}\right), \\
d^{p}\left(f z_{2}, g z_{2}\right), \\
d^{p}\left(f z_{3}, g z_{3}\right)
\end{array}\right\}\right) \leqslant \psi\left(\max \left\{\begin{array}{l}
d^{p}\left(f z_{1}, g z_{1}\right), \\
d^{p}\left(f z_{2}, g z_{2}\right), \\
d^{p}\left(f z_{3}, g z_{3}\right)
\end{array}\right\}\right)-\phi\left(\max \left\{\begin{array}{l}
d^{p}\left(f z_{1}, g z_{1}\right), \\
d^{p}\left(f z_{2}, g z_{2}\right), \\
d^{p}\left(f z_{3}, g z_{3}\right)
\end{array}\right\}\right) .
$$

By using the definition of $\phi$ and $\psi$ we conclude that

$$
\max \left\{\mathrm{d}^{\mathrm{p}}\left(\mathrm{f} z_{1}, \mathrm{~g} z_{1}\right), \mathrm{d}^{\mathrm{p}}\left(\mathrm{f} z_{2}, \mathrm{~g} z_{2}\right), \mathrm{d}^{\mathrm{p}}\left(\mathrm{f} z_{3}, \mathrm{~g} z_{3}\right)\right\}=0,
$$

which implies that

$$
\mathrm{d}^{\mathrm{p}}\left(\mathrm{f} z_{1}, \mathrm{~g} z_{1}\right)=\mathrm{d}^{\mathrm{p}}\left(\mathrm{f} z_{2}, \mathrm{gz} z_{2}\right)=\mathrm{d}^{\mathrm{p}}\left(\mathrm{f} z_{3}, \mathrm{~g} z_{3}\right)=0
$$

Thus

$$
f z_{1}=g z_{1}, \quad f z_{2}=g z_{2}, \quad f z_{3}=g z_{3} .
$$

By putting $x=z_{1}, y=z_{2}, z=z_{3}$ and $u=u_{n}, v=v_{n}, w=w_{n}$ in inequality (2.1) we get

$$
\psi\left(H^{\mathrm{p}}\left(\mathrm{F}\left(z_{1}, z_{2}, z_{3}\right), \mathrm{G}\left(\mathrm{u}_{n}, v_{n}, w_{n}\right)\right)\right) \leqslant \psi\left(\Theta\left(z_{1}, z_{2}, z_{3}, u_{n}, v_{n}, w_{n}\right)\right)-\phi\left(\Theta\left(z_{1}, z_{2}, z_{3}, u_{n}, v_{n}, w_{n}\right)\right),
$$

where,

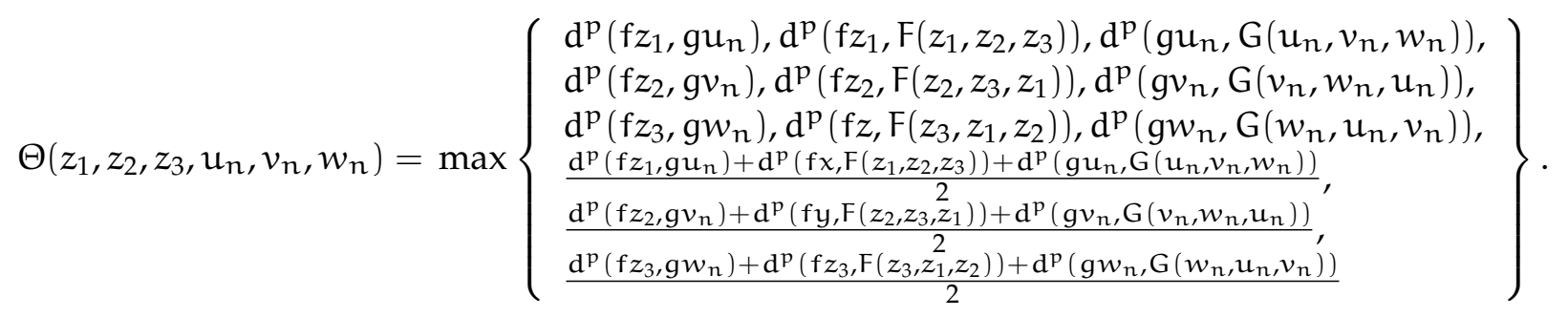

Applying limit to (2.9) and using (2.8) we have

$$
\begin{aligned}
\psi\left(\mathrm{d}^{\mathrm{p}}\left(\mathrm{f} z_{1}, \mathrm{~F}\left(z_{1}, z_{2}, z_{3}\right)\right)\right) \leqslant & \psi\left(\max \left\{\begin{array}{l}
\mathrm{d}^{\mathrm{p}}\left(\mathrm{f} z_{1}, \mathrm{~F}\left(z_{1}, z_{2}, z_{3}\right)\right), \\
\mathrm{d}^{\mathrm{p}}\left(\mathrm{f} z_{2}, \mathrm{~F}\left(z_{2}, z_{3}, z_{1}\right)\right), \\
\mathrm{d}^{\mathrm{p}}\left(\mathrm{f} z_{3}, \mathrm{~F}\left(z_{3}, z_{1}, z_{2}\right)\right)
\end{array}\right\}\right) \\
& -\phi\left(\max \left\{\begin{array}{l}
\mathrm{d}^{\mathrm{p}}\left(\mathrm{f} z_{1}, \mathrm{~F}\left(z_{1}, z_{2}, z_{3}\right)\right), \\
\mathrm{d}^{\mathrm{p}}\left(\mathrm{f} z_{2}, \mathrm{~F}\left(z_{2}, z_{3}, z_{1}\right)\right), \\
\mathrm{d}^{\mathrm{p}}\left(\mathrm{f} z_{3}, \mathrm{~F}\left(z_{3}, z_{1}, z_{2}\right)\right)
\end{array}\right\}\right) .
\end{aligned}
$$

By putting $x=z_{2}, y=z_{3}, z=z_{1}$ and $u=u_{n}, v=v_{n}, w=w_{n}$ in inequality (2.1), by following similar step we get

$$
\begin{aligned}
\psi\left(\mathrm{d}^{\mathrm{p}}\left(\mathrm{f} z_{2}, \mathrm{~F}\left(z_{2}, z_{3}, z_{1}\right)\right)\right) \leqslant & \psi\left(\max \left\{\begin{array}{l}
\mathrm{d}^{\mathrm{p}}\left(\mathrm{f} z_{1}, \mathrm{~F}\left(z_{1}, z_{2}, z_{3}\right)\right), \\
\mathrm{d}^{\mathrm{p}}\left(\mathrm{f} z_{2}, \mathrm{~F}\left(z_{2}, z_{3}, z_{1}\right)\right), \\
\mathrm{d}^{\mathrm{p}}\left(\mathrm{f} z_{3}, \mathrm{~F}\left(z_{3}, z_{1}, z_{2}\right)\right)
\end{array}\right\}\right) \\
& -\phi\left(\left(\max \left\{\begin{array}{l}
\mathrm{d}^{\mathrm{p}}\left(\mathrm{f} z_{1}, \mathrm{~F}\left(z_{1}, z_{2}, z_{3}\right)\right), \\
\mathrm{d}^{\mathrm{p}}\left(\mathrm{f} z_{2}, \mathrm{~F}\left(z_{2}, z_{3}, z_{1}\right)\right), \\
\mathrm{d}^{\mathrm{p}}\left(\mathrm{f} z_{3}, \mathrm{~F}\left(z_{3}, z_{1}, z_{2}\right)\right)
\end{array}\right\}\right) .\right.
\end{aligned}
$$


By placing $x=z_{3}, y=z_{1}, z=z_{2}$ and $u=u_{n}, v=v_{n}, w=w_{n}$ in inequality (2.1), by following same line we have

$$
\begin{aligned}
\psi\left(\mathrm{d}^{\mathrm{p}}\left(\mathrm{f} z_{3}, \mathrm{~F}\left(z_{3}, z_{1}, z_{2}\right)\right)\right) \leqslant & \psi\left(\max \left\{\begin{array}{l}
\mathrm{d}^{\mathrm{p}}\left(\mathrm{f} z_{1}, \mathrm{~F}\left(z_{1}, z_{2}, z_{3}\right)\right), \\
\mathrm{d}^{\mathrm{p}}\left(\mathrm{f} z_{2}, \mathrm{~F}\left(z_{2}, z_{3}, z_{1}\right)\right), \\
\mathrm{d}^{\mathrm{p}}\left(\mathrm{f} z_{3}, \mathrm{~F}\left(z_{3}, z_{1}, z_{2}\right)\right)
\end{array}\right\}\right) \\
& -\phi\left(\max \left\{\begin{array}{l}
\mathrm{d}^{\mathrm{p}}\left(\mathrm{f} z_{1}, \mathrm{~F}\left(z_{1}, z_{2}, z_{3}\right)\right), \\
\mathrm{d}^{\mathrm{p}}\left(\mathrm{f} z_{2}, \mathrm{~F}\left(z_{2}, z_{3}, z_{1}\right)\right), \\
\mathrm{d}^{\mathrm{p}}\left(\mathrm{f} z_{3}, \mathrm{~F}\left(z_{3}, z_{1}, z_{2}\right)\right)
\end{array}\right\}\right) .
\end{aligned}
$$

Combining (2.10), (2.11) and (2.12) we have

$$
\begin{aligned}
\psi\left(\max \left\{\begin{array}{l}
\left(\mathrm{d}^{\mathrm{p}}\left(\mathrm{f} z_{1}, \mathrm{~F}\left(z_{1}, z_{2}, z_{3}\right)\right),\right. \\
\mathrm{d}^{\mathrm{p}}\left(\mathrm{f} z_{2}, \mathrm{~F}\left(z_{2}, z_{3}, z_{1}\right)\right), \\
\mathrm{d}^{\mathrm{p}}\left(\mathrm{f} z_{3}, \mathrm{~F}\left(z_{3}, z_{1}, z_{2}\right)\right)
\end{array}\right\}\right) \leqslant & \psi\left(\max \left\{\begin{array}{l}
\mathrm{d}^{\mathrm{p}}\left(\mathrm{f} z_{1}, \mathrm{~F}\left(z_{1}, z_{2}, z_{3}\right)\right), \\
\mathrm{d}^{\mathrm{p}}\left(\mathrm{f} z_{2}, \mathrm{~F}\left(z_{2}, z_{3}, z_{1}\right)\right), \\
\mathrm{d}^{\mathrm{p}}\left(\mathrm{f} z_{3}, \mathrm{~F}\left(z_{3}, z_{1}, z_{2}\right)\right)
\end{array}\right\}\right) \\
& -\phi\left(\max \left\{\begin{array}{l}
{\left[\mathrm{d}^{\mathrm{p}}\left(\mathrm{f} z_{1}, \mathrm{~F}\left(z_{1}, z_{2}, z_{3}\right)\right),\right.} \\
\mathrm{d}^{\mathrm{p}}\left(\mathrm{f} z_{2}, \mathrm{~F}\left(z_{2}, z_{3}, z_{1}\right)\right) \\
\left.\mathrm{d}^{\mathrm{p}}\left(\mathrm{f} z_{3}, \mathrm{~F}\left(z_{3}, z_{1}, z_{2}\right)\right)\right)
\end{array}\right\}\right),
\end{aligned}
$$

using the definition of $\psi$ and $\phi$ one can get

$$
\max \left\{\begin{array}{l}
d^{p}\left(f z_{1}, F\left(z_{1}, z_{2}, z_{3}\right)\right), \\
d^{p}\left(f z_{2}, F\left(z_{2}, z_{3}, z_{1}\right)\right), \\
d^{p}\left(f z_{3}, F\left(z_{3}, z_{1}, z_{2}\right)\right)
\end{array}\right\}=0,
$$

which implies $d^{\mathfrak{p}}\left(f z_{1}, F\left(z_{1}, z_{2}, z_{3}\right)\right)=0, d^{\mathfrak{p}}\left(f z_{2}, F\left(z_{2}, z_{3}, z_{1}\right)\right)=0$ and $d^{\mathfrak{p}}\left(f z_{3}, F\left(z_{3}, z_{1}, z_{2}\right)\right)=0$. Thus $f z_{1} \in$ $\mathrm{F}\left(z_{1}, z_{2}, z_{3}\right), f z_{2} \in \mathrm{F}\left(z_{2}, z_{3}, z_{1}\right)$ and $\mathrm{f} z_{3} \in \mathrm{F}\left(z_{3}, z_{1}, z_{2}\right)$.

Similarly, by the same procedure one can obtain

$$
g w_{1} \in \mathrm{G}\left(w_{1}, w_{2}, w_{3}\right), \quad g w_{2} \in \mathrm{G}\left(w_{2}, w_{3}, w_{1}\right), \quad g w_{3} \in \mathrm{G}\left(w_{3}, w_{1}, w_{2}\right) .
$$

Since, $f$ is F-weakly commuting so $f^{2} z_{1} \in F\left(f z_{1}, f z_{2}, f z_{3}\right), f^{2} z_{2} \in F\left(f z_{2}, f z_{3}, f z_{1}\right)$ and $f^{2} z_{3} \in F\left(f z_{3} f z_{1}, f z_{2}\right)$. Since, $f^{2} z_{1}=f z_{1}, f^{2} z_{2}=f z_{2}$ and $f^{2} z_{3}=f z_{3}$, thus $\left(f z_{1}, f z_{2}, f z_{3}\right)$ is a common fixed point. A similar argument proves $\left(\mathrm{A}_{4}\right)$. Then using $(2.8)\left(\mathrm{A}_{5}\right)$ holds immediately.

By taking $f(x)=g(x)=x$ in Theorem 2.1 we deduce the following corollary.

Corollary 2.2. Let $\mathrm{F}, \mathrm{G}: \Delta \times \Delta \times \Delta \rightarrow \mathrm{Cl}(\Delta)$ on metric space $(\Delta, \mathrm{d})$, and satisfying the following

$$
\psi\left(H^{p}(F(x, y, z), G(u, v, w))\right) \leqslant \psi(\Theta(x, y, z, u, v, w))-\phi(\Theta(x, y, z, u, v, w)),
$$

where

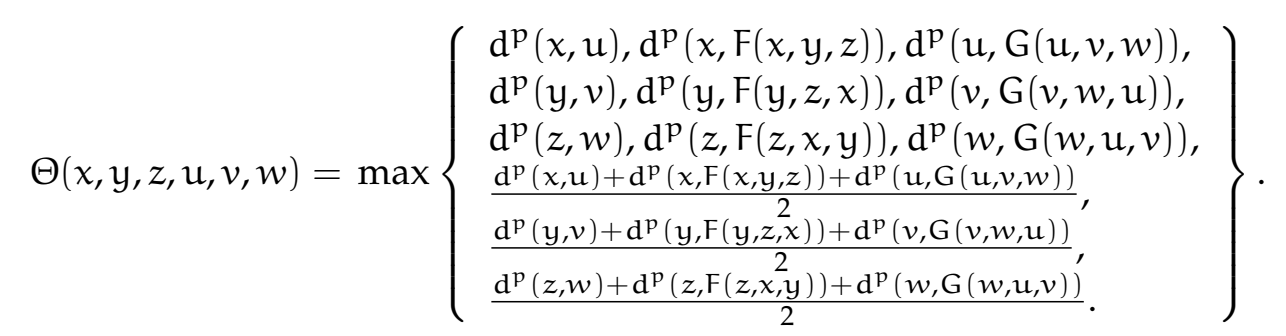

Here, $\psi \in \Psi, \phi \in \Phi$ and $p \geqslant 1$. Then $\mathrm{F}$ and $\mathrm{G}$ have common tripled fixed point.

Similarly, we can prove the following result. 
Theorem 2.3. Let $\mathrm{f}: \Delta \rightarrow \Delta, \mathrm{F}: \Delta \times \Delta \times \Delta \rightarrow \mathrm{Cl}(\Delta)$ on metric space $(\Delta, \mathrm{d})$. Furthermore assume that $(\mathrm{F}, \mathrm{f})$ have $\mathrm{CLR}_{\mathrm{f}}$-property and satisfy the following

$$
\psi\left(H^{p}(F(x, y, z), F(u, v, w)) \leqslant \psi(\Theta(x, y, z, u, v, w))-\phi(\Theta((x, y, z, u, v, w)),\right.
$$

where

$$
\Theta(x, y, z, u, v, w)=\max \left\{\begin{array}{l}
d^{p}(f x, f u), d^{p}(f x, F(x, y, z)), d^{p}(f u, F(u, v, w)), \\
d^{p}(f y, f v), d^{p}(f y, F(y, z, x)), d^{p}(f v, F(v, w, u)), \\
d^{p}(f z, f w), d^{p}(f z, F(z, x, y)), d^{p}(f w, F(w, u, v)), \\
\frac{d^{p}(f x, f u)+d^{p}(f x, F(x, y, z))+d^{p}(f u, F(u, v, w))}{d^{p}(f y, f v)+d^{p}(f y, F(y, z, x))+d^{p}(f v, F(v, w, u))}, \\
\frac{d^{p}(f z, f w)+d^{p}(f z, F(z, x, y))+d^{p}(f w, F(w, u, v))}{2} .
\end{array}\right\} .
$$

Here, $\psi \in \Psi, \phi \in \Phi$ and $\mathrm{p} \geqslant 1$. There exists a common fixed point of $\mathrm{f}$ and $\mathrm{F}$ if one of the following holds:

$\left(A_{1}\right)(F, f)$ are weakly compatible $\lim _{n \rightarrow \infty} f^{n} z_{1}=u, \lim _{n \rightarrow \infty} f^{n} z_{2}=v, \lim _{n \rightarrow \infty} f^{n} z_{3}=w$, for $\left(z_{1}, z_{2}, z_{3}\right) \in C(f, F)$ where $f$ is Continuous at $u, v, w$.

$\left(\mathrm{A}_{2}\right) \mathrm{f}$ is $\mathrm{F}$ weakly commuting $\mathrm{f}^{2} z_{1}=\mathrm{f} z_{1}, \mathrm{f}^{2} z_{2}=\mathrm{f} z_{2}, \mathrm{f}^{2} z_{3}=\mathrm{f} z_{3}$, for $\left(z_{1}, z_{2}, z_{3}\right) \in \mathrm{C}(\mathrm{f}, \mathrm{F})$.

$\left(\mathrm{A}_{3}\right) \lim _{z_{3} \rightarrow \infty} f^{n} u=z_{1}, \lim _{n \rightarrow \infty} f^{n} u=z_{2}, \lim _{n \rightarrow \infty} f^{n} \mathfrak{w}=z_{3}$, for $\left(z_{1}, z_{2}, z_{3}\right) \in C(f, F)$ where $f$ is Continuous at $z_{1}, z_{2}$ and $\left(A_{4}\right) f(C(f, F))$ is singleton set of $C(f, F)$.

Proof. Following the line of the proof of Theorem 2.1, we conclude that $\left(\mathrm{A}_{2}\right)$ holds. Further, we have to check that $\left(A_{1}\right),\left(A_{3}\right)$ and $\left(A_{4}\right)$ hold respectively. Suppose condition $\left(A_{1}\right)$ holds i.e., $\lim _{n \rightarrow \infty} f^{n} z_{1}=u$, $\lim _{n \rightarrow \infty} f^{n} z_{2}=v, \lim _{n \rightarrow \infty} f^{n} z_{3}=w$. Since $f$ is Continuous at $u, v$ and $w$ we have

$$
\mathrm{fu}=\mathrm{u}, \quad \mathrm{fv}=v, \quad \mathrm{fw}=\mathfrak{w} .
$$

As $(F, f)$ are weakly compatible, therefore for $n \geqslant 1$

$$
\left\{\begin{array}{l}
f^{n} z_{1} \in F\left(f^{n-1} z_{1}, f^{n-1} z_{2}, f^{n-1} z_{3}\right), \\
f^{n} z_{2} \in F\left(f^{n-1} z_{2}, f^{n-1} z_{1}, f^{n-1} z_{3}\right), \\
f^{n} z_{3} \in F\left(f^{n-1} z_{3}, f^{n-1} z_{2}, f^{n-1} z_{1}\right)
\end{array}\right\} .
$$

Next,

$$
\begin{aligned}
\psi\left(D^{p}\left(f^{n} z_{1}, F(u, v, w)\right) \leqslant\right. & \psi\left(H^{p}\left(F\left(f^{n-1} z_{1}, f^{n-1} z_{2}, f^{n-1} z_{3}\right), F(u, v, w)\right)\right) \\
\leqslant & \psi\left(\Theta\left(f^{n-1} z_{1}, f^{n-1} z_{2}, f^{n-1} z_{3}, u, v, w\right)\right) \\
& -\phi\left(\Theta\left(f^{n-1} z_{1}, f^{n-1} z_{2}, f^{n-1} z_{3}, u, v, w\right)\right)
\end{aligned}
$$

where

$$
\Theta\left(\left(f^{n-1} z_{1}, f^{n-1} z_{2}, f^{n-1} z_{3}, u, v, w\right)\right)=\max \left\{\begin{array}{l}
d^{p}\left(f^{n} z_{1}, f u\right), d^{p}\left(f^{n} z_{1}, F\left(f^{n-1} z_{1}, f^{n-1} z_{2}, f^{n-1} z_{3}\right)\right), \\
d^{p}(f u, F(u, v, w)), d^{p}\left(f^{n} z_{2}, f v\right), \\
d^{p}\left(f^{n} z_{2}, F\left(f^{n-1} z_{1}, f^{n-1} z_{2}, f^{n-1} z_{3}\right)\right), d^{p}(f v, F(v, w, u)), \\
d^{p}\left(f^{n} z_{3}, f w\right), d^{p}\left(f^{n} z_{1}, F\left(f^{n-1} z_{1}, f^{n-1} z_{2}, f^{n-1} z_{3}\right)\right), \\
d^{p}(f w, F(w, u, v)), \\
\frac{d^{p}\left(f^{n} z_{1}, f u\right), d^{p}\left(f^{n} z_{1}, F\left(f^{n-1} z_{1}, f^{n-1} z_{2}, f^{n-1} z_{3}\right)+d^{p}(f u, F(u, v, w))\right.}{2^{2}}, \\
\frac{d^{p}\left(f^{n} z_{2}, f v\right), d^{p}\left(f^{n} z_{2}, F\left(f^{n-1} z_{2}, f^{n-1} z_{3}, f^{n-1} z_{1}\right)\right)+d^{p}(f v, F(v, w, u))}{2}, \\
\frac{d^{p}\left(f^{n} z_{3}, f w\right)+d^{p}\left(f^{n} z_{3}, F\left(f^{n-1} z_{3}, f^{n-1} z_{1}, f^{n-1} z_{2}\right)\right)+d^{p}(f w, F(w, u, v))}{2} .
\end{array}\right\} .
$$


By taking limit in (2.15) and using (2.14) we get

$$
\begin{aligned}
\psi\left(D^{p}(f u, F(u, v, w))\right) \leqslant & \psi\left(\max \left\{\begin{array}{l}
d^{p}(f u, F(u, v, w)), \\
d^{p}(f v, F(v, w, u)), \\
d^{p}(f w, F(w, u, v))
\end{array}\right\}\right) \\
& -\phi\left(\max \left\{\begin{array}{l}
d^{p}(f u, F(u, v, w)), \\
d^{p}(f v, F(v, w, u)), \\
d^{p}(f w, F(w, u, v))
\end{array}\right\}\right) .
\end{aligned}
$$

Similarly we have

$$
\begin{aligned}
\psi\left(D^{p}(f v, F(v, w, u))\right) \leqslant & \psi\left(\max \left\{\begin{array}{l}
d^{p}(f u, F(u, v, w)), \\
d^{p}(f v, F(v, w, u)), \\
d^{p}(f w, F(w, u, v))
\end{array}\right\}\right) \\
& -\phi\left(\max \left\{\begin{array}{l}
d^{p}(f u, F(u, v, w)), \\
d^{p}(f v, F(v, w, u)), \\
d^{p}(f w, F(w, u, v))
\end{array}\right\}\right),
\end{aligned}
$$

and

$$
\begin{aligned}
& \psi\left(D^{p}(f w, F(w, u, v))\right) \leqslant \psi\left(\max \left\{\begin{array}{l}
d^{p}(f u, F(u, v, w)), \\
d^{p}(f v, F(v, w, u)), \\
d^{p}(f w, F(w, u, v))
\end{array}\right\}\right) \\
&-\phi\left(\max \left\{\begin{array}{l}
d^{p}(f u, F(u, v, w)), \\
d^{p}(f v, F(v, w, u)), \\
d^{p}(f w, F(w, u, v))
\end{array}\right\}\right) .
\end{aligned}
$$

Combining (2.16), (2.17), (2.18) in last we get

$$
D^{p}(f u, F(u, v, w))=0, \quad D^{p}(f v, F(v, u, w))=0, \quad D^{p}(f w, F(w, v, w))=0,
$$

which implies $f u \in F(u, v, w), f v \in F(v, u, w)$ and $f w \in F(w, v, w)$. Hence using $(2.13)(u, v, w)$ is a common fixed point of $F$ and $f$.

Suppose $\left(A_{3}\right)$ holds. Assume $\left(z_{1}, z_{2}, z_{3}\right) \in C(f, F)$, then $\lim _{n \rightarrow \infty} f^{n} u=z_{1}, \lim _{n \rightarrow \infty} f^{n} u=z_{2}, \lim _{n \rightarrow \infty} f^{n} w=z_{3}$. Since $f$ is Continuous at $z_{1}, z_{2}$ and $z_{3}$, therefore

$$
f z_{1}=z_{1}, \quad f z_{2}=z_{2}, \quad f z_{3}=z_{3} .
$$

Since $\left(z_{1}, z_{2}, z_{3}\right) \in C(f, F)$, therefore $f z_{1} \in F\left(z_{1}, z_{2}, z_{3}\right), f z_{2} \in F\left(z_{2}, z_{1}, z_{3}\right)$ and $f z_{3} \in F\left(z_{3}, z_{2}, z_{1}\right)$. Hence from (2.19), $\left(z_{1}, z_{2}, z_{3}\right)$ is a common tripled fixed point of $F$ and $f$.

Finally, let $f(C(f, F))=\left(z_{1}, z_{2}, z_{3}\right)$ then $\left\{z_{1}\right\}=\left\{f z_{1}\right\}=F\left(z_{1}, z_{2}, z_{3}\right)$. Hence $\left(z_{1}, z_{2}, z_{3}\right)$ is tripled fixed point of $f$ and $F$.

\section{Applications to systems of integral equations}

On ward, we develop sufficient conditions for the solutions to the following general nonlinear systems of Fredholm integral equations of 2nd kind given by

$$
\begin{cases}\eta_{i}(t)=\phi(t)+\int_{\zeta}^{\sigma} K_{1}\left(f_{i}(t), f_{i+1}(t), f_{i+2}(t)\right) d t, & t \in[\zeta, \sigma], \\ \zeta_{i}(t)=\phi(t)+\int_{\zeta}^{\sigma} K_{2}\left(f_{i}(t), f_{i+1}(t), f_{i+2}(t)\right) d t, & t \in[\zeta, \sigma], \quad i=1,2,3 .\end{cases}
$$


Let $\Lambda=L^{1}[\zeta, \sigma]$ be the set of all Lebesgue integrable functions on $[\zeta, \sigma]$. Define $d: \Lambda \times \Lambda \rightarrow \mathbb{R}^{+}$by

$$
\mathrm{d}(\eta, \zeta)=\int_{\zeta}^{\sigma} \operatorname{l\eta }(x)-\zeta(x) \mid \mathrm{d} x
$$

Then $(\Lambda, d)$ is a metric space on $\Lambda$. For the derivation of aforesaid condition, we give the following theorem.

Theorem 3.1. Assume that the following assumptions holds:

$\left(\mathrm{A}_{1}\right) \phi: \mathbb{R}^{+} \rightarrow \mathbb{R}^{+}$is continuous.

$\left(\mathrm{A}_{2}\right)$ There exist $\mathrm{K}_{1}, \mathrm{~K}_{2}: \mathbb{R}^{3} \rightarrow \mathbb{R}$ which are continuous functions such that

$$
\left|K_{1}\left(x_{i+1}, x_{i+2}, x_{i+3}\right)-K_{2}\left(y_{i+1}, y_{i+2}, y_{i+3}\right)\right| \leqslant \max \left\{\begin{array}{c}
\left|x_{i+1}-y_{i+2}\right|, \\
\left|x_{i+2}-y_{i+2}\right|, \\
\left|x_{i+3}-y_{i+3}\right| .
\end{array}\right\},
$$

for $i=1,2,3$.

Then, the systems of integral equations (3.1) has a common solution in $\mathrm{L}^{1}[\zeta, \sigma]$.

Proof. Define F, G : $\mathrm{L}^{1}[\zeta, \sigma]^{3} \rightarrow \mathrm{L}^{1}[\zeta, \sigma]$ by

$$
\left\{\begin{array}{l}
F_{f_{i+1} f_{i+2} f_{i+3}}(t)=\phi(t)+\int_{\zeta}^{\sigma} K_{1}\left(f_{i}(t), f_{i+1}(t), f_{i+2}(t)\right) d t, \quad t \in[\zeta, \sigma], \\
G_{g_{i+1} g_{i+2} g_{i+3}}(t)=\phi(t)+\int_{\zeta}^{\sigma} K_{2}\left(g_{i}(t), g_{i+1}(t), g_{i+2}(t)\right) d t, \quad t \in[\zeta, \sigma], \quad i=1,2,3 .
\end{array}\right.
$$

Now, we have

$$
\begin{aligned}
d\left(F_{f_{i+1} f_{i+2} f_{i+3}}, G_{g_{i+1} g_{i+2} g_{i+3}}\right) & \\
& =\int_{\zeta}^{\sigma}\left|F_{f_{i+1}, f_{i+2} f_{i+3}}(t)-G_{g_{i+1} g_{i+2} g_{i+3}}(t)\right| d x \\
& =\int_{\zeta}^{\sigma}\left(\int_{\zeta}^{\sigma}\left|k_{1}\left(f_{i+1}(t), f_{i+2}(t), f_{i+3}(t)\right)-k_{2}\left(g_{i+1}(t), g_{i+2}(t), g_{i+3}(t)\right)\right| d t\right) d x \\
& \leqslant \int_{\zeta}^{\sigma}\left(\int_{\zeta}^{\sigma} \max \left\{\left|f_{i+1}(t)-g_{i+1}(t)\right|,\left|f_{i+2}(t)-g_{i+2}(t)\right|,\left|f_{i+3}(t)-g_{i+3}(t)\right|\right\} \mid d t\right) d x .
\end{aligned}
$$

Now, there are three cases for $i=1,2,3$.

If $i=1$ again there are three cases.

Case 1. If

$$
\max \left\{\left|f_{2}(t)-g_{2}(t)\right|,\left|f_{3}(t)-g_{3}(t)\right|,\left|f_{4}(t)-g_{4}(t)\right|\right\}=\left|f_{2}(t)-g_{2}(t)\right|,
$$

then inequality (3.2) implies that

$$
\begin{aligned}
d\left(F_{f_{2} f_{3} f_{4}}, G_{g_{2} g_{3} g_{4}}\right) & \leqslant \int_{\zeta}^{\sigma}\left(\int_{\zeta}^{\sigma}\left|f_{2}(t)-g_{2}(t)\right| d t\right) d x \\
& =\int_{\zeta}^{\sigma}\left(d\left(f_{2}, g_{2}\right)\right) d x \\
& =(\sigma-\zeta)\left(d\left(f_{2}, g_{2}\right)\right) \\
& \leqslant(\sigma-\zeta) \Theta\left(f_{2}, f_{3} f_{4}, g_{2}, g_{3}, g_{4}\right) .
\end{aligned}
$$

Case 2. If

$$
\max \left\{\left|f_{2}(t)-g_{2}(t)\right|,\left|f_{3}(t)-g_{3}(t)\right|,\left|f_{4}(t)-g_{4}(t)\right|\right\}=\left|f_{3}(t)-g_{3}(t)\right|,
$$


then from inequality (3.2) we have

$$
\begin{aligned}
d\left(F_{f_{2} f_{3} f_{4},} G_{g_{2} g_{3} g_{4}}\right) & \leqslant \int_{\zeta}^{\sigma}\left(\int_{\zeta}^{\sigma}\left|f_{3}(t)-g_{3}(t)\right| d t\right) d x \\
& =\int_{\zeta}^{\sigma}\left(d\left(f_{3}, g_{3}\right)\right) d x \\
& =(\sigma-\zeta)\left(d\left(f_{3}, g_{3}\right)\right) \\
& \leqslant(\sigma-\zeta) \Theta\left(f_{2}, f_{3} f_{4}, g_{2}, g_{3}, g_{4}\right) .
\end{aligned}
$$

Case 3. If

$$
\max \left\{\left|f_{2}(t)-g_{2}(t)\right|,\left|f_{3}(t)-g_{3}(t)\right|,\left|f_{4}(t)-g_{4}(t)\right|\right\}=\left|f_{4}(t)-g_{4}(t)\right|,
$$

then from inequality (3.2) we have

$$
\begin{aligned}
d\left(F_{f_{2} f_{3} f_{4}}, G_{g_{2} g_{3} g_{4}}\right) & \leqslant \int_{\zeta}^{\sigma}\left(\int_{\zeta}^{\sigma}\left|f_{4}(t)-g_{4}(t)\right| d t\right) d x \\
& =\int_{\zeta}^{\sigma}\left(d\left(f_{4}, g_{4}\right)\right) d x \\
& =(\sigma-\zeta)\left(d\left(f_{4}, g_{4}\right)\right) \\
& \leqslant(\sigma-\zeta) \Theta\left(f_{2}, f_{3} f_{4}, g_{2}, g_{3}, g_{4}\right) .
\end{aligned}
$$

Similar result can be held for other two cases for $i=2,3$. By taking $\psi(\eta)=(\sigma-\zeta) \eta, \phi(\eta)=0, p=1$ from Corollary 2.2 the system of integral equations (3.1) has common solution in the form of $\left(f_{0}, f_{0}, f_{0}\right)$.

\section{Acknowledgment}

This work was supported by the National Natural Science Foundation of China (11571378).

The authors are also grateful to the editor and referees for their comments and remarks to improve this manuscript.

\section{References}

[1] A. A. N. Abdou, Common fixed point results for multi-valued mappings with some examples, J. Nonlinear Sci. Appl., 9 (2016), 787-798. 1

[2] Y. I. Alber, S. Guerre-Delabriere, Principle of weakly contractive maps in Hilbert spaces, New results in operator theory and its applications, Oper. Theory Adv. Appl., Birkhäuser, Basel, 98 (1997), 7-22. 1

[3] A. Amini-harandi, D. O'Regan, On coupled and tripled fixed point theory of multi-valued contraction mappings in partially ordered metric spaces, Commun. Appl. Anal., 19 (2015), 209-216. 1

[4] H. Aydi, M. Abbas, Tripled coincidence and fixed point results in partial metric spaces, Appl. Gen. Topol., 13 (2012), 193-206. 1

[5] V. Berinde, M. Borcut, Tripled fixed point theorems for contractive type mappings in partially ordered metric spaces, Nonlinear Anal., 74 (2011), 4889-4897. 1

[6] B. Deshpande, C. Kothari, A. Handa, Common tripled fixed point theorems under weaker conditions, Int. J. Pure Appl. Math., 103 (2015), 1-17. 1

[7] B. Deshpande, S. Sharma, A. Handa, Tripled fixed point theorem for hybrid pair of mappings under generalized nonlinear contraction, J. Korean Soc. Math. Educ. Ser. B Pure Appl. Math., 21 (2014), 23-38. 1, 1.3, 1.4, 1.5

[8] D. Doric, Common fixed point for generalized ( $\psi, \phi)$-weak contractions, Appl. Math. Lett., 22 (2009), 1896-1900. 1

[9] P. N. Dutta, B. S. Choudhury, A generalisation of contraction principle in metric spaces, Fixed Point Theory Appl., 2008 (2008), 8 pages. 1

[10] T. Gnana Bhaskar, V. Lakshmikantham, Fixed point theorems in partially ordered metric spaces and applications, Nonlinear Anal., 65 (2006), 1379-1393. 1

[11] J. Harjani, K. Sadarangani, Generalized contractions in partially ordered metric spaces and applications to ordinary differential equations, Nonlinear Anal., 72 (2010), 1188-1197. 1 
[12] M. A. Khan, Sumitra, CLRg property for coupled xed point theorems in fuzzy metric spaces, Int. J. Appl. Phys. Math., 2 (2012), 355-358. 1

[13] V. Lakshmikantham, L. Ćirić, Coupled fixed point theorems for nonlinear contractions in partially ordered metric spaces, Nonlinear Anal., 70 (2009), 4341-4349. 1

[14] S. B. Nadler Jr., Multi-valued contraction mappings, Pacific J. Math., 20 (1969), 475-488. 1.11, 1.12, 1

[15] K. P. R. Rao, G. N. V. Kishore, K. Tas, A unique common triple fixed point theorem for hybrid pair of maps, Abstr. Appl. Anal., 2012 (2012), 9 pages. 1

[16] K. P. R. Rao, K. V. Siva Parvathi, M. Imdad, hybrid coupled fixed point theorems for maps under (CLRg) property in fuzzy metric spaces, Novi Sad J. Math., (2015), 18 pages. 1

[17] K. P. R. Rao, A. Sombabu, M. Mustaq Ali, Common coupled fixed point theorems satisfying (CLRg) property in complex valued b-metric spaces, Bull. Int. Math. Virtual Inst., 6 (2016), 189-198. 1

[18] B. E. Rhoades, Some theorems on weakly contractive maps, Proceedings of the Third World Congress of Nonlinear Analysts, Part 4, Catania, (2000), Nonlinear Anal., 47 (2001), 2683-2693. 1

[19] B. Samet, C. Vetro, Coupled fixed point, F-invariant set and fixed point of N-order, Ann. Funct. Anal., 1 (2010), 46-56. 1.2

[20] W. Shatanawi, M. Postolache, Z. Mustafa, Tripled and coincidence fixed point theorems for contractive mappings satisfying Ф-maps in partially ordered metric spaces, An. Ştiinţ. Univ. "Ovidius" Constanţa Ser. Mat., 22 (2014), 179-203. 1

[21] W. Sintunavarat, P. Kumam, Common fixed point theorems for a pair of weakly compatible mappings in fuzzy metric spaces, J. Appl. Math., 2011 (2011), 14 pages. 1.6

[22] Q.-N. Zhang, Y.-S. Song, Fixed point theory for generalized $\phi$-weak contractions, Appl. Math. Lett., 22 (2009), 75-78. 1 